\title{
Agaricoid basidiomycetes of the natural park Uch-Enmek of Altai Republic
}

\author{
Irina Gorbunova* \\ Central Siberian Botanical Garden SB RAS, 630090, Novosibirsk, RussianFederation
}

\begin{abstract}
The data on locations, habitats and vegetation season of 205 species agaricoid basidiomycetes new for the Karakol natural park UchEnmek (Altai Republic) are presented. They were collected in the steppe, forest, forest-steppe and highland belts. Among them 32 species are recorded for the first time for Altai Republic, 6 species are rare in Altai Republic and Western Siberia.
\end{abstract}

The Karakol natural park Uch-Enmek is a specially protected area in the Ongudai region of the Altai Republic. It was established in 2001 by a decree of the Government of the Altai Republic. There are many cultural and historical monuments of different time periods and locations sacred for the Altai population in the park.

The Karakol natural park Uch-Enmek is located in the Central Altai province of the Altai mountain region. A significant part of its territory is confined to the valley of the river Karakol. It includes the near-valley slopes and mountain ranges of the northern spurs of the Terekta ridge. A significant variety of relief, a large amplitude of absolute heights (over $1800 \mathrm{~m}$ ) that causes changes in the ratio of heat and moisture to height, a clearly pronounced exposure contributes to the formation of high-altitude-zonal complexes (alpine, mountain-forest, forest-steppe exposition type, mountain-steppe).

Alpine landscapes (1) are represented by the tundra belt (dryad, fescue and dwarf birch tundras) (a), alpine and subalpine meadows (b), cedar and larch open forest (c), tundra bogs (d). More than half of the area of the park is occupied by mountain-forest landscapes (2), represented by larch-cedar, cedar-larch (a), spruce-cedar-larch forests (b), growing on mountain-forest brown soils. Forest-steppe landscapes (3) gravitate towards the steep slopes of southern exposures on rubble-loamy soils. The vegetation cover is dominated by wormwood-grass and fescue-feather grass dry steppes (a). Higher on the slopes, shrubgrass steppes (b) and their petrophytic variants (c) dominate on infantile mountain-steppe soils. Larch, birch-larch forests (d) on mountain-forest, in places dark gray soils are formed on the slopes of the northern exposures. The valleys of "small" rivers are occupied by spruce, larch-spruce forests with areas of tall grass meadows (4) on alluvial humus-gley soils [1]. On the territory of the park, there are anthropogenic habitats (pastures, plowing, etc.) (5).

A systematic study of the biota of macromycetes in the Altai Republic during the entire growing season in various plant belts was carried out for the first time on the territory of the

* Corresponding author: fungi2304@gmail.com 
Karakol natural park Uch-Enmek in 2010-2011. Collection and herbarization of the material was conducted according to the standard method [2], mainly in the vicinity of the village Kulada and the lake Arukem. Identification of herbarium samples was carried out in the laboratory of mycology, algology and lichenology of the Central Siberian Branch of the SB RAS (Novosibirsk) using a "Primo Star" microscope Carl Zeiss Jena, a standard set of reagents and dyes [3], and modern mycological literature. The collection is stored in the Herbarium of M.G. Popov of the CSBG SB RAS, Novosibirsk (NSK). Presented below is the list of species of agaricoid basidiomycetes identified in the Uch-Enmek Natural Park. The taxonomy and nomenclature of the species complies with the electronic resource "Index Fungorum" (www.indexfungorum.org) (date of access 17 VI 2021) [4]. The list contains the name of the species, as well as the habitat (in Arabic numerals) and the growing season (in Roman numerals) noted in brackets. The new species for the Altai Republic are marked with the * symbol, rare species for Altai Republic and Western Siberia are marked with the ** symbol.

\section{Agaricales}

Agaricaceae: Agaricus campestris L. (3a, 5, VII-VIII), A. comtulus Fr. (3d, VII), Cystoderma amianthinum (Scop.) Fayod (4, VII-VIII) C. cinnabarina (Alb. et Schwein.) Harmaja (3d, VII-IX), Cystodermella granulosa (Batsch) Harmaja (2b, 3d, VIII), Lepiota clypeolaria (Bull.) P. Kumm. (3d, VIII), L. erminea (Fr.) Gillet (3a, 3d, VII-VIII), *L. pseudolilacea Huijsman (3d, VII), *L. subincarnata J.E. Lange (3d, VII), **Leucoagaricus nympharum (Kalchbr.) Bon (3d, VIII), Macrolepiota campestris Lebedeva ex Samgina (3a, VIII), M. excoriata (Schaeff.) Wasser (3a,b,d, VIII).

Amanitaceae: Amanita crocea (Quél.) Singer (4, VII), A. nivalis Grev. (1a, VII-VIII), A. submembranacea (Bon) Gröger (1c, VII-VIII), Limacella glioderma (Fr.) Maire (2a, VIII).

Bolbitiaceae: Bolbitius lacteus J.E. Lange (4, VIII), B. titubans (Bull.) Fr. (1b, 3d, 5, V-IX), Conocybe arrhenii (Fr.) Kits van Wav. (1c, VIII), ${ }^{*}$ C. mutabilis (Watling) Bon (4, VII), C. siennophylla (Berk. et Broome) Singer (3d, 5, VIII), C. blattaria (Fr.) Kühner (2a, b, VIII), C. velata (Velen.) Watling (4, VI), C. vexans P.D. Orton (4, VIII).

Cortinariaceae: Cortinarius armillatus (Fr.) Fr. (2b, VIII), C. balaustinus Fr. (2b, VIII), [5], C. collinitus (Sowerby) Gray (2b, VII) [5], C. caperatus (Pers.) Fr. (1a, VIII), C. croceus (Schaeff.) Gray (2b, VIII), C. decipiens (Pers.) Fr. (4, VIII), C. scaurus (Fr.) Fr. (2b, VIII) [5], $* C$. vespertinus (Fr.) Fr. (2b, VIII), *C. torvus (Fr.) Fr. (2b, VIII), C. porphyropus (Alb. et Schwein.) Fr. (2b, VIII).

Entolomataceae: Rhodocybe caelata (Fr.) Maire (3d, 2b, VIII), R. nitellina (Fr.) Singer (2b, VIII), Entoloma clypeatum (L.) P. Kumm. (3a, VII), E. rhodopolium (Fr.) P. Kumm. (3d, VIII).

Hygrophoraceae: Arrhenia oniscus (Fr.) Redhead, Lutzoni, Moncalvo et Vilgalys (2b, VII), Cantharellula umbonata (J.F. Gmel.) Singer (2b, VIII), Hygrocybe psittacina (Schaeff.) P. Kumm. (3d, VIII), Hygrophorus lucorum Kalchbr. (2a, VII-VIII), ${ }^{*} H$. speciosus Peck (2b, VII-VIII), Lichenomphalia alpina (Britzelm.) Redhead, Lutzoni, Moncalvo et Vilgalys (4, VI), L. umbellifera (L.) Redhead, Lutzoni, Moncalvo et Vilgalys $(1,2,4, \mathrm{~V}-\mathrm{X})$.

Hydnangiaceae: Laccaria altaica Singer (56 VII), L. bicolor (Maire) P.D. Orton (1a, 1b, VII-VIII), L. laccata (Scop.) Cooke (2a, 4, VIII), *L. pseudomontana Osmundson, C. Cripps et G.M. Muell. (1d, VII), L. pumila Fayod (4, VIII).

Hymenogastraceae: Galerina hypnorum (Schrank) Kühner (4, VIII), G. marginata (Batsch) Kühner (2a, VIII), G. pumila (Pers.) M. Lange (2a, VIII), G. sphagnicola (G.F. Atk.) A.H. Sm. et Singer (2b, VII), **Gymnopilus flavus (Bres.) Singer (3c, VII), G. penetrans (Fr.) Murrill (2a, VIII), G. sapineus (Fr.) Murrill (2a, VIII), Hebeloma birrus (Fr.) Sacc. (2b, VIII), *H. fusisporum Gröger et Zschiesch. (1c, VII), H. helodes J. Favre 
(2b, VIII), H. crustuliniforme (Bull.) Quél. (4, VIII), H. kuehneri Bruchet (2b, VIII), H. leucosarx P.D. Orton (4c, VIII), H. mesophaeum (Pers.) Quél. (4c, VIII), *H. monticola Vesterh. (2b, VII).

Inocybaceae: Inocybe appendiculata Kühner (3d, VII), I. dulcamara (Pers.) P. Kumm. (3d, VII), I. geophylla (Sowerby) P. Kumm. (1c, 2b, VII-VIII), I. godeyi Gillet (3d, VII), I. griseolilacina J.E. Lange (1c, VIII), *I. leptocystis G.F. Atk (4, VIII), I. nematoloma Joss. (4, VIII), I. ochroalba Bruyl. (3d, VII), I. petiginosa (Fr.) Gillet (2b,VII), I. sambucina (Fr.) Quél (2b, VII) [5], I. whitei (Berk. et Broome) Sacc. (4, VII), Simocybe sumptuosa (P.D. Orton) Singer (2b, VIII).

Lyophyllaceae: Rugosomyces chrysenteron (Bull.) Bon (3d, VII), Tephrocybe rancida (Fr.) Donk (4, VIII).

Macrocystidiaceae: Macrocystidia cucumis (Pers.) Joss. (2a, VIII).

Marasmiaceae: Marasmius epiphyllus (Pers.) Fr. (2a, VIII), Marasmius oreades (Bolton) Fr. (3c, VII), Crinipellis scabella (Alb. et Schwein.) Murrill (4, VII).

Omphalotaceae: Gymnopus confluens (Pers.) Antonín, Halling et Noordel. (4, VII), Rhodocollybia maculata (Alb. et Schwein.) Singer (2b, VIII).

Mycenaceae: Hemimycena delectabilis (Peck) Singer (3d, VIII), Mycena aetites (Fr.) Quél. (4, VI-VII), M. acicula (Schaeff.) P. Kumm. (4, VI), *M. clavicularis (Fr.) Gillet (2b, VIII), *Mycena cinerella (P. Karst.) P. Karst. (3d, VII-VIII), M. citrinomarginata Gillet (1a, 1b, VIII), M. epipterygia (Scop.) Gray (2b, VIII), M. galericulata (Scop.) Gray (2, 3, 4, VII-X), M. haematopus (Pers.) P. Kumm. (4, VI-VII), M. hiemalis (Osbeck) Quél. (4, VIVII), M. laevigata Gillet (1c, 2, 3, 4, VII-VIII), M. leptocephala (Pers.) Gillet (2, 3, 4, VII$\mathrm{X})$, M. filopes (Bull.) P. Kumm. (4, VI-VII), *M. flavescens Velen. (4, VIII), M. maculata P. Karst. (2a, 2b, VIII), M. metata (Secr. ex Fr.) P. Kumm. (3d, VII), M. olivaceomarginata (Massee) Massee (2, 3, 4, VII-VIII), M. pura (Pers.) P. Kumm. (2, 3d, 4, VI-X), M. purpureofusca (Peck) Sacc. (2a, VII), Mycena rubromarginata (Fr.) P. Kumm. (2, 3, 4, VII-VIII), M. strobilicola J. Favre et Kühner (4, V), M. viridimarginata P. Karst. (4, VIIVIII), *M. urania (Fr.) Quél. (2b, VII), Xeromphalina cauticinalis (With.) Kühner et Maire $(2,3,4, \mathrm{~V}-\mathrm{X})$.

Phyllotopsidaceae: Phyllotopsis nidulans (Pers.) Singer (4, 5, VII).

Physalacriaceae: Armillaria borealis Marxm. et Korhonen (4, VIII), *A. obscura (Schaeff.) Herink (4, VIII), Flammulina velutipes (Curtis) Singer (4, VII).

Pleurotaceae: *Pleurotus dryinus (Pers.) P. Kumm. (2a, VIII), P. ostreatus (Jacq.) P. Kumm. (2, 3, 4, VIII).

Pluteaceae: Pluteus cervinus (Schaeff.) P. Kumm. (2a, VIII), P. leoninus (Schaeff.) P. Kumm. (2a, VI), P. nanus (Pers.) P. Kumm. (2a, VIII), *P. pouzarianus Singer (4, VIII), $P$. umbrosus (Pers.) P. Kumm. (2b, VIII), Volvopluteus gloiocephalus (DC.) Vizzini, Contu et Justo (3d, 5, VIII).

Psathyrellaceae: *Coprinellus marculentus (Britzelm.) Redhead, Vilgalys et Moncalvo (3d, VIII), *Coprinopsis ephemeroides (DC.) G. Moreno (1c, , VII), C. nivea (Pers.) Redhead, Vilgalys et Moncalvo (5, VII-X), Psathyrella candolleana (Fr.) Maire (2a, VIII), *P. phegophila Romagn. (4, VII), *P. pertinax (Fr.) Örstadius (2a, VIII), P. prona (Fr.) Gillet (4, VI-VII), P. pseudogracilis (Romagn.) M.M. Moser (4, VI-VIII).

Pseudoclitocybaceae: Bonomyces sinopicus (Fr.) Vizzini (= Clitocybe sinopica (Fr.) P. Kumm.) (2a, VIII).

Strophariaceae: *Hypholoma polytrichi (Fr.) Ricken (2b, VIII), H. lateritium (Schaeff.) P. Kumm. (2, 3d, 4, V-VIII), Kuehneromyces lignicola (Peck) Redhead (4, VII), Pholiota flammans (Batsch) P. Kumm. (2a, VIII), P. squarrosa (Vahl) P. Kumm. (2a, VIII), P. spumosa (Fr.) Singer (4, VIII), Deconica merdicola (Huijsman) Noordel. (3a,b,c, 4. VIIX), Protostropharia semiglobata (Batsch) Redhead, Moncalvo et Vilgalys (1, 2, 3, 4, VI$\mathrm{X})$. 
Tricholomataceae: *Aspropaxillus giganteus (Sowerby) Kühner et Maire (3a, VII), Leucopaxillus paradoxus (Costantin et L.M. Dufour) Boursier (2b, 1c, VII-VIII), Tricholoma fulvum (Fr.) Bigeard et H. Guill. (4, VIII), T. lascivum (Fr.) Gillet (2a, VIII), T. stiparophyllum (S. Lundell) P. Karst. (2a,b, VIII), **T. odorum Peck (2a, VIII), T. terreum (Schaeff.) P. Kumm. (3d, VII), T. vaccinum (Schaeff.) P. Kumm. (4, VIII), T. virgatum (Fr.) P. Kumm. (2b, VIII).

Tubariaceae: *Flammulaster carpophilus (Fr.) Earle ex Vellinga (4, VII), F. muricata (Fr.) Watling (5, VII), Tubaria conspersa (Pers.) Fayod (4, VIII), T. furfuracea (Pers.) Gillet $(2,3,4, \mathrm{~V}-\mathrm{X}),{ }^{* *}$ T. minutalis Romagn. (3a, VII).

Incertae sedis: *Baeospora myriadophylla (Peck) Singer (4, VIII), Clitocybe fragrans (With.) P. Kumm. (3d, VIII), Collybia cirrhata (Schumach.) Quél. (2a, VII-VIII), Infundibulicybe geotropa (Bull.) Harmaja (2a, VIII), Lepista flaccida (Sowerby) Pat. (2a, VIII), L. densifolia (J. Favre) Singer et Clémençon (4, VIII), Melanoleuca grammopodia (Bull.) Murrill (2a, VIII), M. graminicola (Velen.) Kühner et Maire (2a, VII), M. strictipes (P. Karst.) Jul. Schäff. (1a, VII), Tricholomopsis decora (Fr.) Singer (4, VIII), Panaeolus ater (J.E. Lange) Kühner et Romagn. (1a, VI), P. fimicola (Pers.) Gillet (2, 3d, 4, 5, VII$\mathrm{X})$, P. semiovatus (Sowerby) S. Lundell et Nannf. (3a,d,c. 4, 5, V-X), Lignomphalia lignicola (Lj.N. Vassiljeva) Antonín, Borovička, Holec et Kolařík (= Pseudoomphalina lignicola Lj.N. Vassiljeva) (2a, VII VIII).

\section{Boletales}

Boletaceae: Leccinum scabrum (Bull.) Gray (4, VIII).

Gomphidiaceae: Gomphidius maculatus (Scop.) Fr. (3d, VIII).

Suillaceae: Boletinus asiaticus Singer (4, VIII), *B. spectabilis Peck (4, VIII), *Suillus bovinus (Pers.) Roussel (2b, VIII), *S. bresadolae Cazzoli et Consiglio (2b, 4, VIII), S. cavipes (Opat.) A.H. Sm. et Thiers (= Boletinus cavipes (Opat.) Kalchbr.) (3d, VI-VIII), *S. flavus (With.) Singer (3d, VIII), S. grevillei (Klotzsch.) Singer (1a, 2a, 3d, VI-X), S. sibiricus (Singer) Singer (2b, VIII), **S. tridentinus (Bres.) Singer (2a, 4, VIII-X), S. viscidus (L.) Roussel (1c, 2a,b, 3d, 4, VI-VIII).

Paxillaceae: Paxillus involutus (Batsch) Fr. (2a, VIII).

Tapinellaceae: *Tapinella panuoides (Batsch) E.-J. Gilbert (2a, VIII).

\section{Russulales}

Russulaceae : Lactarius deterrimus Gröger (4, VIII), L. dryadophilus Kühner (1a, VII), L. duplicatus A.H. Sm. (2a, X), *L. fulvissimus Romagn. (2a, X), *L. leonis Kytöv. (4, VIII), L. porninsis Rolland (2a, 3d, VIII-X), L. pubescens (Fr.) Fr (4, VIII), L. rufus (Scop.) Fr. (2a, VIII), L. subdulcis (Pers.) Gray (4, VIII), L. trivialis (Fr.) Fr. (2b, VIII), L. turpis (Weinm.) Fr. (2d, X), Russula aeruginea Fr. (2a,b, VII), R. cyanoxantha (Schaeff.) Fr. (4, VIII), $R$. delica Fr. (2b, 4, VII-VIII), R. nana Killerm. (1a, VIII), R. queletii Fr. (2a, 3d, 4, VII-VIII), *R. paludosa Britzelm. (2b, VII), R. puellaris Fr. (2a, 4, VIII), $R$. ochroleuca (Pers.) Fr. (1a, VII), *Russula pallidospora J. Blum ex Romagn. (1a, VII), *R. subrubens (J.E. Lange) Bon (R. chamiteae Kühner) (1a, VII), R. xerampelina (Schaeff.) Fr. (2b, VII).

\section{References}

1. G. S. Samoilova, World of Science, Culture, Education, 2 (14) (2009)

2. A. S. Bondartsev, R. A. Zinger, Proceedings of the Botanical Institute. V. L. Komarova, Series II, 6 (1950)

3. H. Clemenson, Methods for working with macrofungi (IHW-Verl., Eching, 2009)

4. Index Fungorum (www.indexfungorum.org) (date of the application 17 VI 2021)

5. I. A. Gorbunova, Yu. A. Rebriev, Plant Life of Asian Russia, 3 (35) (2019) 